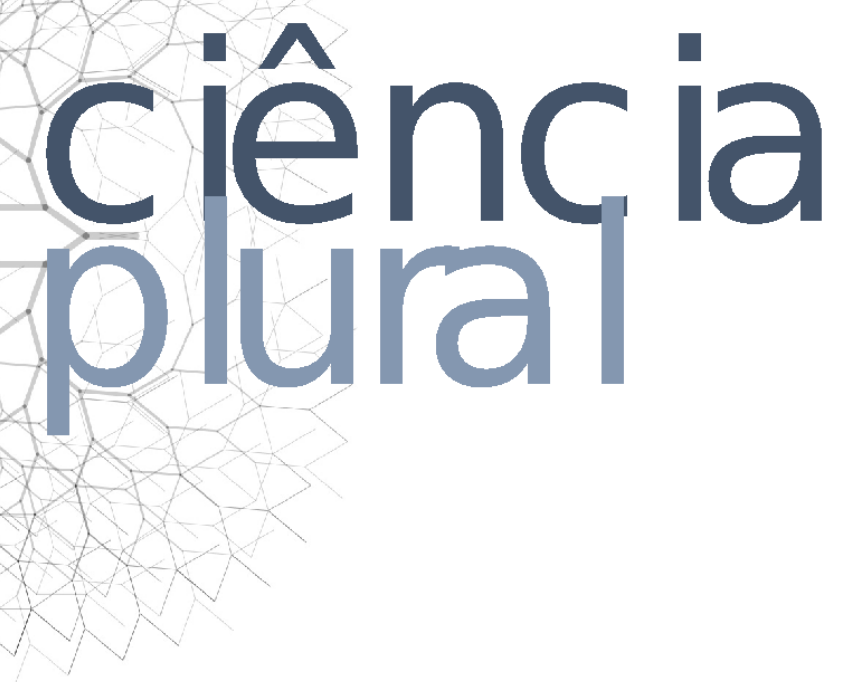

\title{
O IMPACTO DA COVID-19 NA SAÚDE DE PESSOAS INSTITUCIONALIZADAS
}

The impact of COVID-19 on the health of institutionalized persons

El impacto del COVID-19 em la salude de las personas institucionalizada

Pedro Duarte Ferreira Neto • aluno de graduação em Odontologia • Universidade Federal do Rio Grande do Norte-UFRN • E-mail: pedro_duarte_25@yahoo.com.br

Clara Wilma Fernandes Rosendo • aluna de graduação em Medicina • UFRN • E-mail: clararondes@hotmail.com

Flávio Anselmo Silva de Lima • aluno de graduação em Saúde Coletiva • UFRN • E-mail: flavio_ifrn@hotmail.com

Yanka Patrícia Ferreira Bezerra - Enfermeira - Universidade Potiguar • E-mail: yankapatricia-@outlook.com

Sulamita Pereira da Silva Lima • aluna de graduação em Saúde Coletiva • UFRN • E-mail: sulaesuele@hotmail.com

Vilani Medeiros de Araújo Nunes • Professora Associada do Departamento de Saúde Coletiva • UFRN・E-mail: vilani.nunes@ufrn.br

Autor correspondente:

Pedro Duarte Ferreira Neto E-mail: pedro_duarte_25@yahoo.com.br 


\section{RESUMO}

Introdução: A população idosa constitui um dos grupos mais vulneráveis à infecção pelo novo coronavírus. Tal fragilidade é ainda mais evidente naqueles que residem em Instituições para Idosos, por apresentar maior risco de contaminação e, ainda, de morbimortalidade. Objetivo: Identificar o impacto da COVID-19 na saúde de pessoas idosas residentes nas Instituições de Longa Permanência. Metodologia: Trata-se de estudo retrospectivo de revisão integrativa da literatura, entre os meses de novembro/2020 e março/2021, sintetizando estudos publicados sobre a temática. A partir do objetivo traçado para este estudo, determinamos quais seriam as questões norteadoras: 1. "Quais medidas estão sendo adotadas para minimizar os efeitos da COVID-19 nas Instituições de Longa Permanência para Idosos?" 2. "Quais os principais impactos causados por essas medidas?". Estes eixos colaboram para a compreensão dos acontecimentos de significativa relevância social. Resultados: As instituições para idosos, como ambientes coletivos, possuem residentes com elevada vulnerabilidade à infecção pelo novo coronavírus. Naqueles idosos acometidos por quadros de demência e outras doenças neurológicas, o isolamento social aprofunda a gravidade da infecção pela COVID-19, dificultando, assim, as atividades relacionadas à atenção e assistência realizadas pelos cuidadores. Ademais, caracteriza-se como medida fundamental a adoção do rastreamento laboratorial precoce para potencializar a prevenção de casos e promover o controle da infecção. Percebe-se ainda uma deficiência referente à construção de um protocolo com medidas de profilaxia e manejo adequado de modo mais direcionado dentro dessas instituições. Conclusão: Torna-se urgente um olhar mais atento às necessidades da população idosa, especialmente do grupo institucionalizado, de modo a promover políticas de investimento em cuidados de saúde integrais e realizados por equipe multiprofissional.

Palavras-Chave: COVID-19; Idoso; Instituição de Longa Permanência Para Idosos.

\section{BSTRACT}

oduction: The elderly population is one of the groups most vulnerable to infection by the new coronavirus. Such fragility is even more evident in those who live in Institutions for the Aged, as it presents a higher risk of contamination and, even, of morbidity and mortality. Objective: To identify the impact of COVID-19 on the health of elderly people residing in Long Term Institutions for the Aged. Methodology: This is a retrospective study of integrative literature review, between the months of November/2020 and March/2021, synthesizing published studies on the theme. Based on the objective outlined for this study, we determined what the guiding questions would be: 1 . "What measures are being taken to minimize the effects of COVID-19 on Long Term Care Institutions for the Aged?" 2. "What are the main impacts caused by these measures?. These axes contribute to the understanding of events of significant social relevance. Results: Institutions for the aged, as collective environments, have with high vulnerability to infection with the new coronavirus. In those aged 
people affected by dementia and other neurological diseases, social isolation deepens the severity of the infection by COVID-19, thus hampering the activities related to the care and assistance performed by caregivers. In addition, the adoption of early laboratory screening is characterized as a fundamental measure to enhance the prevention of cases and promote infection control. It is also perceived a deficiency regarding the construction of a protocol with prophylaxis measures and adequate management in a more targeted way within these institutions. Conclusions: It is urgent to look more closely at the needs of the aged population, especially the institutionalized group, in order to promote investment policies in comprehensive health care carried out by a multidisciplinary team.

Key words: COVID-19; Aged; Long Term Institution for the Aged.

\section{RESUMEN}

Introducción: la población anciana es uno de los grupos más vulnerables a la infección por el nuevo coronavirus. Tal fragilidad es aún más evidente en quienes viven en Instituciones de Ancianos, ya que presenta un mayor riesgo de contaminación e, incluso, de morbilidad y mortalidad. Objetivo: Identificar el impacto del COVID-19 en la salud de los ancianos que residen en Instituciones de Atención de Larga Duración. Metodología: Se trata de un estudio retrospectivo de revisión integradora de la literatura, entre los meses de noviembre/2020 y marzo/2021, sintetizando los estudios publicados sobre el tema. Con base en el objetivo delineado para este estudio, determinamos cuáles serían las preguntas orientadoras: 1. "¿Qué medidas se están tomando para minimizar los efectos del COVID-19 en las Instituciones de Atención de Larga Duración para el Anciano?" 2. “¿Cuáles son los principales impactos provocados por estas medidas?". Estos ejes contribuyen a la comprensión de hechos de relevancia social significativa. Resultados: Las instituciones para los ancianos, como entornos colectivos, tienen residentes con alta vulnerabilidad a la infección por el nuevo coronavirus. Aquellos ancianos afectados por demencia y otras enfermedades neurológicas, el aislamiento social profundiza la gravedad de la infección por COVID19, dificultando así las actividades relacionadas con el cuidado y asistencia que realizan los cuidadores. Además, la adopción del cribado precoz de laboratorio se caracteriza por ser una medida fundamental para potenciar la prevención de casos y promover el control de infecciones. También se percibe una deficiencia en la construcción de un protocolo con medidas de profilaxis y manejo adecuado de manera más focalizada dentro de estas instituciones. Conclusiones: Es urgente mirar más de cerca las necesidades de la población anciana, especialmente del grupo institucionalizado, para promover políticas de inversión en la atención integral de salud llevadas a cabo por un equipo multidisciplinario.

clave: COVID-19; Anciano; Instituciones de Atención de Larga Duración nciano. 


\section{Introdução}

A COVID-19 é uma síndrome respiratória aguda, causada pelo coronavírus SARS-CoV-2, potencialmente grave, de elevada transmissibilidade e de distribuição global que vem causando preocupação e pânico em todo o mundo. Os idosos representam a parcela da população dentre os mais vulneráveis para o risco de desenvolver a forma grave da doença, podendo levar ao óbito.

Segundo dados do Ministério da Saúde, no período de janeiro a março de 2021, o maior número de óbitos por Covid-19 ocorre na população idosa, ou seja, de pessoas a partir dos 60 anos, com cerca de 22.887 óbitos, apresentando um número bastante expressivo quando comparado com a soma das outras faixas etárias neste período (7.418), sendo a faixa etária maior de 60 anos a mais susceptíveis por apresentarem diversos fatores de risco, tais como hipertensão arterial, diabetes mellitus e doenças cardiovasculares, que, usualmente, ocorrem de forma associada, agravando o quadro da infecção pelo novo coronavírus $1,2,3$.

Considerando o elevado número de idosos que residem em Instituições de Longa Permanência (ILPI), essas vêm se tornando um dos ambientes onde os cuidados devem ser redobrados para evitar a contaminação dos que nelas residem. Tais centros consistem no principal fator de risco para morbimortalidade entre idosos, visto que apresenta um conjunto de fatores críticos para o aumento da contaminação e da fragilidade na faixa etária analisada. $4,5,6$.

Pode-se compreender o impacto da infecção por esse novo patógeno entre idosos residentes em ILPI ao se verificar a taxa de transmissibilidade do vírus nessas instituições, a qual supera $60 \%$ e gera consequências devastadoras de elevada mortalidade, especialmente entre grupos de idosos com mais comorbidades 5,6.

Fonsiderando o elevado número de pessoas idosas que residem em ILPI, e stes domicílios coletivos, seus residentes são extremamente vulneráveis à tendo um forte impacto na população que reside nessas instituições 7,8 . 
O quadro de maior vulnerabilidade associada ao envelhecimento está relacionado à significativa frequência de declínio da capacidade funcional, de modo a gerar sérios impactos na fragilidade e dependência na população de pessoas idosas, assim como associa-se ao processo natural de senescência advinda com a idade 4,10,11.

No contexto das IPLI, a maior tendência de aproximação com outros indivíduos idosos, bem como com cuidadores e outros profissionais, somada à influência exercida pela existência de doenças crônicas cardiovasculares, metabólicas, neurológicas e demais, contribuem para um contexto desfavorável à prevenção e promoção de saúde ao idoso.

Sendo assim, a contaminação pelo novo coronavírus resulta em um panorama desfavorável, em especial para pessoas idosas, de modo a aumentar o risco de complicações graves, como necessidade de internamentos, comprometimento preocupante da capacidade funcional e, em casos mais agravantes, risco potencial de mortalidade. Tal situação mostra-se ainda mais nítida entre pessoas idosas portadoras de patologias crônicas, as quais potencializam a fragilidade dessa população 12,13.

Nessa perspectiva, o objetivo do estudo é identificar o impacto da COVID-19 na saúde de pessoas idosas residentes de Instituições de Longa Permanência.

\section{Tetodologia}

Trata-se de estudo retrospectivo de revisão integrativa da literatura, por reunir estudos científicos já produzidos e permitir a avaliação da temática estudada e enriquecer a produção científica com uma temática atual e relevante. A metodologia do estudo foi baseada no que recomenda a metodologia PRISMA (Preferred Reporting Itens for Systematic Reviews and Meta-analyses) com pesquisa realizada na cidade de Natal (Rio Grande do Norte, na região do Nordeste brasileiro) no período compreendido entre Novembro de 2020 e Março de 2021, sintetizando estudos publicados sobre a temática. A partir do objetivo traçado para este estudo, determinamos quais seriam as questões norteadoras, a partir de dois eixos de 
discussão: 1. "Quais medidas estão sendo adotadas para minimizar os efeitos da COVID-19 na ILPI?" 2. "Quais são os principais impactos causados por essas medidas?". Estes eixos colaboram para a compreensão dos acontecimentos de significativa relevância social.

Para realizar a seleção dos artigos, seguiu-se um processo dividido em quatro etapas. A etapa 1 consistiu da verificação do número de artigos encontrados nas bases de dados selecionadas e leitura dos títulos dos artigos selecionados com o pretexto de excluir os textos que não atendiam aos critérios de elegibilidade; para a etapa 2, foi realizada mais uma leitura dos títulos para eliminação dos artigos duplicados; na etapa 3, após a leitura dos resumos dos artigos selecionados foram eliminados os estudos que não atendiam aos critérios de inclusão, então segui-se com a avaliação dos artigos restantes, através da leitura completa do texto e nova eliminação daqueles que não atendiam aos critérios de inclusão; na etapa 4, foi realizada a síntese das informações evidenciadas nos artigos selecionados, nesta etapa, ainda, foram extraídos os dados de todos os artigos selecionados.

\section{Estratégia de busca}

As bases de dados selecionadas foram: Scientific Electronic Library Online (Scielo), Literatura Latino Americana e do Caribe em Ciências da Saúde (Lilacs) e Medical Literature Analysis and Retrieval System Online (Medline). Adotou-se, em todas as bases de dados, a modalidade "busca avançada", por meio de combinação variável entre os descritores nos idiomas português e inglês "idoso", "COVID-19" e “Instituição de Longa Permanência para Idosos" ("idoso" AND “COVID-19” AND “Instituição de Longa Permanência para Idosos”).

Realizada a busca nas três bases, foram encontrados 16 estudos, após aplicados os critêrios de inclusão, considerando o intervalo de publicação de Fevereiro de 2020 a Março de 2021. A escolha do período se deu em virtude do surgimento da doença no ano de 2020 no território brasileiro, com a persistente disseminação da doença até os dias atuais. Além disso, há a possibilidade de estudar seus impactos em vários aspectos da população brasileira, em especial do grupo das pessoas idosas, as quais 
são altamente afetadas pelos efeitos trágicos da doença no corpo humano. Ao final, foram selecionados 4 artigos para a construção do trabalho, após aplicados os critérios de exclusão.

\section{Critérios de elegibilidade dos estudos}

Em nossa busca, consideramos como critérios de inclusão artigos gratuitos, disponibilizados integralmente e que correspondem à temática analisada no estudo. Os artigos analisados foram lidos e seus tópicos foram organizados em uma tabela em Word, separados por cada grupo ("nome do artigo", "autores", "objetivos", "metodologia", resultados" e "conclusão"). Os critérios de exclusão foram: notas técnicas, artigos duplicados e não coerentes com o objetivo buscado.

A partir dos artigos que atenderam aos critérios de elegibilidade propostos, foram extraídas as seguintes características: a) título do artigos, autores e ano de publicação do estudo; b) objetivos do estudo selecionado; c) instrumentos empregados na pesquisa; d) metodologia empregada.

\section{Seleção dos estudos}

Após a pesquisa nas bases de dados, realizou-se uma triagem inicial com base nos títulos dos estudos, excluindo os trabalhos não coerentes com a proposta de nossa investigação. Feita a leitura dos resumos, os artigos que não atenderam aos critérios de elegibilidade foram excluídos. Portanto, os resultados apresentados em nosso estudo são a síntese dos artigos selecionados para análise. Todo o processo de seleção foi realizado de forma independente por dois pesquisadores, sendo as discordâncias mínimas existentes resolvidas por consenso.

O estudo não necessitou de submissão ao Comitê de Ética em Pesquisa (CEP), por se tratar de utilização de base de dados secundários, conforme rege a Resolução nº510/2016 do Conselho Nacional de Saúde. 


\section{Resultados}

A partir da busca na literatura, foram identificados 11 artigos no LILACS, 7 no MEDLINE e 2 no SciELO, resultando em um total de 20 estudos. Após aplicarmos os critérios de exclusão aos estudos encontrados, 16 foram eliminados. Assim, 4 artigos foram utilizados para análise. Todas as etapas de seleção dos estudos estão detalhadas na Figura 1. Os 4 artigos incluídos foram avaliados qualitativamente quanto ao tipo do estudo, seus objetivos e resultados alcançados. Esses dados são mostrados na tabela 1. Os artigos selecionados para análise não apresentaram o mesmo desfecho e possuíam características diferentes, a exemplo do grupo amostral, faixa etária e outros parâmetros para avaliação. A partir do quadro 1, observa-se que todos os estudos foram publicados em 2020, ou seja, não houve a publicação de novos artigos, que contemplassem os objetivos deste estudo, no período analisado (Fevereiro/2020 a Março/2021).

Foi identificado que as Instituições de Longa Permanência para idosos, como ambientes coletivos, possuem residentes com elevada vulnerabilidade à infecção pelo novo coronavírus. Nos idosos residentes que são acometidos por quadros de demência, delirium e outras doenças neurológicas, o efeito do isolamento social acaba confundindo a gravidade da infecção pela COVID-19, dificultando, assim, as atividades relacionadas à atenção e assistência prestadas pelos cuidadores 8 . 
Figura 1. Etapas cumpridas pelo PRISMA para seleção dos artigos. Natal/RN, 2021.

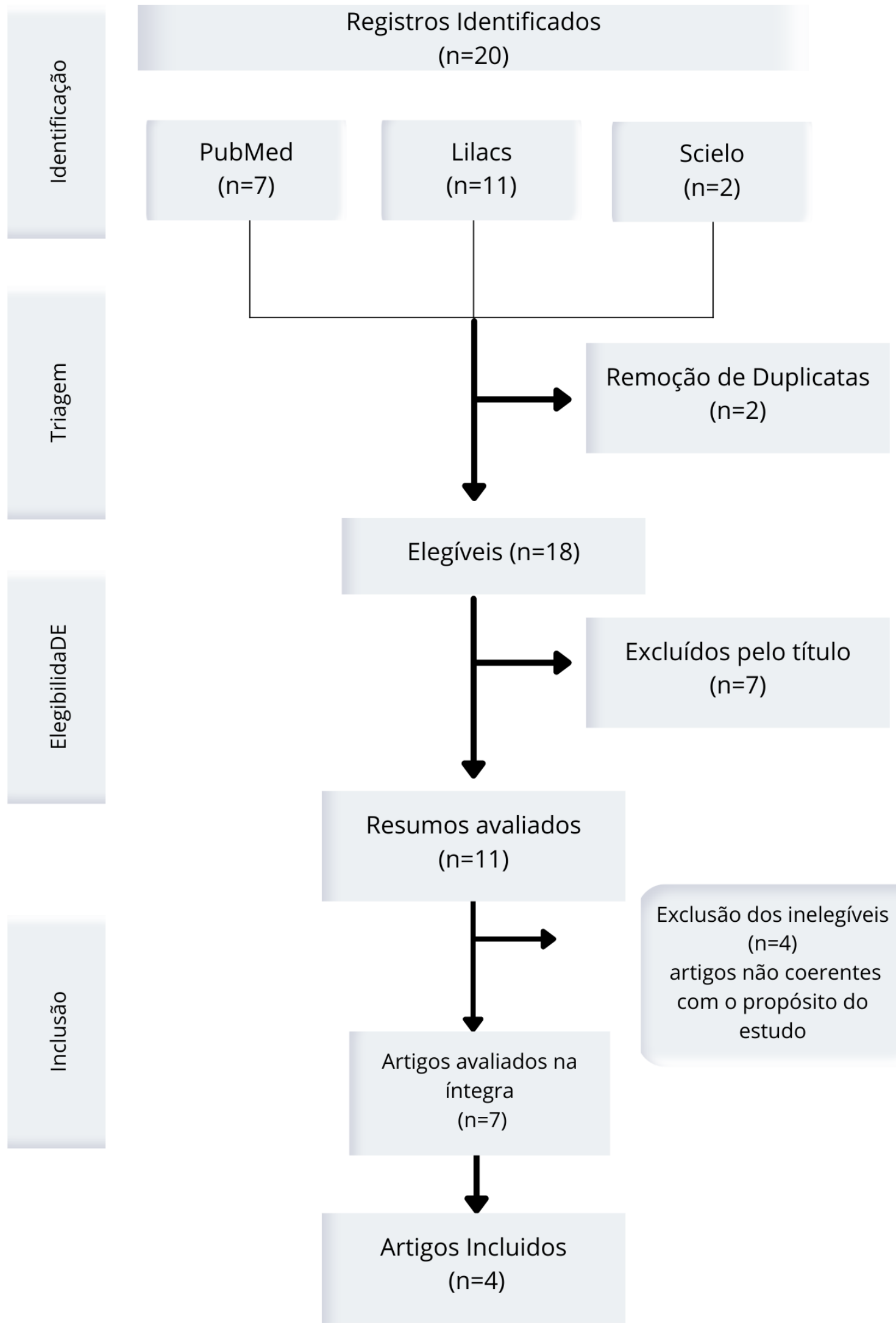


Tabela 1. Aspectos gerais dos artigos selecionados para elaboração da revisão integrativa. Natal/RN, 2021.

\begin{tabular}{|c|c|c|c|}
\hline $\begin{array}{l}\text { TÍTULO DO } \\
\text { ARTIGO, AUTORES } \\
\text { E ANO }\end{array}$ & OBJETIVOS & METODOLOGIA EMPREGADA & RESULTADOS \\
\hline $\begin{array}{c}\text { COVID-19 nas } \\
\text { instituições de longa } \\
\text { permanência para } \\
\text { idosos: } \\
\text { estratégias de } \\
\text { rastreamento } \\
\text { laboratoriale } \\
\text { prevenção da } \\
\text { propagação da doença } \\
\text { Moraes EN et al., } 2020\end{array}$ & $\begin{array}{l}\text { Propõe-se uma estratégia de } \\
\text { rastreamento e } \\
\text { monitoramento das } \\
\text { Instituições de Longa } \\
\text { Permanência para Idosos para } \\
\text { mitigar a transmissão do } \\
\text { SARS-CoV-2. }\end{array}$ & $\begin{array}{l}\text { Foi realizada uma pesquisa dos diferentes meios de detecção } \\
\text { da doença causada por COVID-19 e alguns dos detalhes } \\
\text { técnicos, histórico e valores de referências associados aos } \\
\text { testes estudados. }\end{array}$ & $\begin{array}{l}\text { Os idosos institucionalizados representam o } \\
\text { principal alvo da infecção pelo SARS-CoV-2, } \\
\text { com altas taxas de mortalidade. O } \\
\text { rastreamento laboratorial de idosos residentes } \\
\text { e trabalhadores de ILPI é uma estratégia de } \\
\text { controle e prevenção que deve ser associada às } \\
\text { outras medidas protetivas, de forma sinérgica. } \\
\text { Os testes imunológicos, dentre eles os TLR ou } \\
\text { testes rápidos, podem ser utilizados como } \\
\text { alternativas factíveis e complementares no } \\
\text { rastreamento laboratorial da COVID-19, } \\
\text { dependendo do acesso das ILPI brasileiras a } \\
\text { tais opções. }\end{array}$ \\
\hline $\begin{array}{c}\text { Estimativas de } \\
\text { impacto da COVID-19 } \\
\text { na mortalidade de } \\
\text { idosos } \\
\text { institucionalizados no } \\
\text { Brasil } \\
\text { Machado CJ et al, } 2020\end{array}$ & $\begin{array}{l}\text { Estimar o impacto da } \\
\text { COVID-19 na mortalidade de } \\
\text { idosos institucionalizados no } \\
\text { Brasil. }\end{array}$ & $\begin{array}{l}\text { Trata-se de estudo transversal e de simulação. As estimativas } \\
\text { apresentadas neste trabalho foram calculadas em duas } \\
\text { etapas. Na primeira etapa, estimaram-se os óbitos totais em } \\
\text { idosos por COVID-19 e na segunda, aplicou-se a esses óbitos } \\
\text { uma porcentagem ponderada obtida com base em } \\
\text { indicadores de mortalidade proporcional advindas de dados } \\
\text { oficiais de óbitos em instituições de longa permanência para } \\
\text { idosos de diferentes países - como China, Itália e Coréia do } \\
\text { Sul - ao Brasil. }\end{array}$ & $\begin{array}{l}\text { Estimaram-se } 107.538 \text { óbitos de idosos nestas } \\
\text { instituições no Brasil em } 2020 \text {, por COVID-19. } \\
\text { São previstos maiores números de óbitos na } \\
\text { Região Sudeste ( } 48.779 \text { óbitos), seguida da } \\
\text { Região Nordeste ( } 28.451 \text { óbitos); São Paulo é a } \\
\text { Unidade da Federação que na estimativa será } \\
\text { mais afetada ( } 24.500 \text { óbitos). }\end{array}$ \\
\hline
\end{tabular}

Recomendações para o enfrentamento da disseminação da COVID-19 em

Instituições de Longa

Permanência para

Idosos

Santana RF et al, 2020

Telemonitoramento a instituições de longa permanência para idosos frente às infecções por coronavirus e COVID-19

Menezes TMO et al 2020
Elaborar um protocolo de Estudo de recomendações de especialistas utilizando-se um $\mathrm{O}$ protocolo foi estruturado em um núcleo de recomendaçōes para o formulário estruturado aplicado por meio da Técnica Delphi, intervençōes de enfermagem para o enfrentamento da obtendo-se $100 \%$ de concordância entre os profissionais após enfrentamento da disseminação da COVID-19 disseminação quatro rodadas de análise. A população foi constituída por em Instituições de Longa Permanência para da COVID-19 em Instituições seis enfermeiros membros do Departamento Científico de Idosos, consistindo em 8 ações. de Longa Permanência para Enfermagem Gerontológica da Associação Brasileira de Idosos.
Enfermagem

Relatar a experiência de Relato descritivo da experiência ocorrida entre 18 de março e Foram acompanhadas 32 instituições durante telemonitoramento de 25 de abril de 2020, através de telemonitoramento das quatro semanas e algumas facilidades e Instituições de Longa instituições de Salvador, $\mathrm{Ba}$, seguindo um roteiro dificuldades se apresentaram no decorrer do Permanência para Idosos previamente elaborado para primeiro contato e de monitoramento. frente às infecções por seguimento. $\mathrm{O}$ telemonitoramento foi realizado por docentes coronavírus e COVID-19. da Escola de Enfermagem da Universidade Federal da Bahia e discentes do Programa de Pós-Graduação dessa escola, durante quatro semanas. 
Com o intuito de desacelerar, ou até mesmo interromper a doença dentro das ILPI, devem ser adotadas boas práticas que preservem a vida das pessoas idosas residentes nessas instituições. Assim, uma das melhores formas de barrar a contaminação seria a identificação precoce dos indivíduos infectados a fim de adotar medidas que interrompam o ciclo de transmissão local ${ }^{4}$. Essas medidas podem ser adotadas a partir de protocolos que identifiquem práticas a serem adotadas na prevenção e controle da doença ${ }^{9}$.

Além da construção de medidas de prevenção e controle torna-se essencial um olhar atento às necessidades de saúde emocional da população idosa, especialmente residentes em ILPI, tendo em vista o frequente isolamento e distanciamento social presentificados nesse grupo, mesmo antes da pandemia. Assim, deve-se entender o sério impacto exercido pela pandemia na saúde mental dessas pessoas, cujo resultado foi o agravamento da condição emocional e piora do distanciamento social nas Instituições.

Nessa situação, estudos realizados dentro de tal abordagem pontuam um comprometimento mais sério proporcionado pela pandemia da COVID-19 diretamente na saúde psicológica das pessoas idosas, haja vista que esse grupo é mais suscetível aos efeitos negativos do isolamento social e ao medo de contaminação pelo vírus. Além disso, tais pesquisas consolidam a necessidade urgente de investimentos públicos pelas autoridades políticas, de modo a amenizar as consequências drásticas na saúde mental e produzir melhorias no cuidado ao grupo de pessoas idosas 14,15. Pode-se ainda avaliar a condição de pessoas idosas com deficiências neurocognitivas, as quais potencializam os sintomas psicossomáticos agravados pelo isolamento social, como agitação, ansiedade e afastamento do convívio social, impactando gravemente, dessa forma, a preservação da saúde física e mental dos indivíduos idosos ${ }^{16}$.

Nesse sentido, sinaliza-se a construção de protocolos como recomendação importante, no sentido de fornecer para cuidadores e profissionais da saúde um entendimento mais amplo de como realizar o diagnóstico e manejo de indivíduos idosos residentes em ILPI. A partir desses documentos, torna-se possível a adoção de 
uma conduta mais eficaz quanto ao tratamento adequado dos portadores da doença, bem como quanto à profilaxia de pessoas vulneráveis ao risco de infecção.

Sob esse contexto é pertinente a disseminação ampla do protocolo feito pela Sociedade Brasileira de Geriatria e Gerontologia e intitulado “Recomendações para Prevenção e Controle de Infecções por Coronavírus (SARS-Cov-2) em Instituições de Longa Permanência para Idosos (ILPI)". Tal documento possui o papel de contribuir para a compressão e facilitar o manejo das pessoas idosas desses centros por parte da equipe multiprofissional de saúde, em parceria com os cuidadores das ILPI. Além de orientar sobre prevenção e controle, esse protocolo recomenda a adoção de planos individuais de acordo com as necessidades de cada Instituição, de modo a proporcionar ações de profilaxia e vigilância adaptadas a cada realidade ${ }^{17}$.

Aliado ao material supracitado, outros documentos foram construídos de modo a ampliar o conhecimento das medidas de controle e prevenção da doença e, assim, diminuir o impacto gerado pela pandemia. Assim, a cartilha nomeada "COVID-19 e o cuidado de idosos: Recomendações para Instituições de Longa Permanência" foi construída visando-se propor recomendações de medidas protetivas e de controle para o manejo de pessoas idosas residentes em ILPI. Esse documento segue as orientações propostas pela Organização Mundial da Saúde (OMS), pelo Ministério da Saúde (MS) e da Agência Nacional de Vigilância Sanitária (ANVISA) ${ }^{18}$.

Ademais, em face do potencial elevado de morbimortalidade pela COVID-19 nas Instituições de Longa Permanência, deve ser realizada pesquisa epidemiológica que, além de notificar a quantidade de infecções de de óbitos decorrentes da nova doença, analise quais são os fatores inerentes a esse contexto, de modo a gerar impactos positivos sobre a qualidade de vida dos indivíduos idosos, bem como permitir a redução da disseminação da doença nesses centros ${ }^{19}$.

O impacto gerado pela pandemia pela COVID-19 nas Instituições de Longa Permanência ainda é uma temática pouco discutida e publicada em estudos. Em razão de tal contexto, é coerente a realização de pesquisas mais aprofundadas que estudem 
o perfil de vulnerabilidade associada ao envelhecimento, especialmente quando relacionado à infecção pela COVID-19.

\section{Conclusões}

Considerando o elevado número de idosos vulneráveis em nosso país, a COVID-19 proporciona um impacto significativo e preocupante na saúde de pessoas idosas residentes de ILPI, visto que esse grupo configura um dos segmentos sociais mais atingidos pela nova doença, com elevados índices de morbidade e mortalidade. Sendo assim, as pessoas idosas são gravemente comprometidas pelo risco associado à disseminação intensa do novo coronavírus e, devido a isso, faz-se necessária a ampliação de medidas de combate ao contexto analisado. Tal ação pode ser feita mediante a criação e fortalecimento de políticas públicas de auxílio às ILPI são necessárias, tendo em vista as necessidades de diversos recursos estruturais e humanos para o enfrentamento da doença. Diante do exposto, torna-se necessário um olhar mais atento para as ILPI, na perspectiva de desenvolver políticas públicas que priorizem o cuidado precoce e as condutas com medidas protetivas, bem como tratamento eficaz e individualizado para as pessoas idosas, por parte da equipe de cuidado multidisciplinar das referidas instituições. Sugere-se ainda enfatizar a importância da inclusão da temática de saúde da pessoa idosa nos cursos de graduação da área da saúde das universidades, no sentido de gerar formação de futuros profissionais com conhecimentos direcionados a atenção a essa faixa etária em crescimento no país.

\section{rências}

1 Boletim Epidemiológico Especial 8 [Internet]. Ministério da Saúde, Secretaria de Vigilância em Saúde. Brasil. 2021 [cited 2021 april 13]. Available from: https:/ /www.gov.br/saude/pta/pdf/2021/marco/05/boletim_epidemiologico_covid_52_final2.pdf. 
o enfrentamento da COVID-19 em Uberlândia, Minas Gerais. APS em Revista 2020; 2(1):38-43.

3 Guan W, Ni Z, Hu Y, Liang W, Ou C, He J, et al. China Medical Treatment Expert Group for Covid-19. Clinical Characteristics of Coronavirus Disease 2019 in China. N Engl J Med 2020; 382(18):1708-1720.

4 Moraes EN, Viana LG, Resende LMH, Vasconcellos LS, Moura AS, Menezes A, Mansano NH, Rabelo R. COVID-19 nas instituições de longa permanência para idosos: estratégias de rastreamento laboratorial e prevenção da propagação da doença. Ciência \& Saúde Coletiva. 2020, 25(9): 3445-58.

5 Arons MM, Hatfield KM, Reddy SC, Kimball A, James A, Jacobs JR, Taylor J, Spicer K, Bardossy AC, Oakley LP. Presymptomatic SARS-CoV-2 Infections and Transmission in a Skilled Nursing Facility. N Engl J Med. 2020; 382(22): 2081-2090.

6 Gandhi M, Yokoe DS, Havlir DV. Asymptomatic Transmission, the Achilles' Heel of Current Strategies to Control Covid-19. N Engl J Med. 2020; 382(22): 2158-60.

7 Machado CJ, Pereira CCA, Viana BM, Oliveira GL, Melo DC, Carvalho JFMG, Moraes FL, Moraes EN. Estimativas de impacto da COVID-19 na mortalidade de idosos institucionalizados no Brasil. Ciência \& Saúde Coletiva. 2020; 25(9): 3437-44.

8 Menezes TMO, Freitas AVS, Pedreira LC, Amaral JB. Telemonitring of Brazilian Nursing homes before Coronavirus and COVID-19 Infections. Rev. Bras. Enferm. [online]. 2020, 73(Suppl. 2): 1-5 e20200350.

9 Santana RF, Silva MB, Marcos DASR, Rosa CS, Junior WDW, Delvalle R. Nursing recommendations for facing dissemination of COVID-19 in Brazilian Nursing Homes. Revista Brasileira de Enfermagem. 2020; 73(Suppl. 2): 1-9 e20200260.

10 D'adamo H, Yoshikawa T, Ouslander JG. Coronavirus Disease 2019 in Geriatrics and Long-Term Care: the abcds of covid-19. J Am Geriatr Soc. 2020; 68:912-7

11 Fallon A, Dukelow T, Kennelly SP, O'Neill D. COVID-19 in nursing homes. Qjm: An International Journal of Medicine, 2020, 113(6):391-2

12 Center for Disease Control (CDC) [Internet]. “Coronavirus Disease 2019 (COVID19)". Centers for Disease Control and Prevention. 11 de fevereiro de 2020. [Updated Dec. 13, 2020; cited 2020 Dez 17]. Available from: https:/ /www.cdc.gov/coronavirus/2019-ncov/specific-groups/high-risk- 
13 Andrew M, Searle SD, McElhaney JE, McNeil SA, Clarke B, Rockwood K, Kelvin DJ. COVID-19, frailty and long-term care: Implications for policy and practice. J Infect Dev Ctries. 2020; 14(5):428-432.

14 Alves A, Magalhães I. Implicações na saúde mental de idosos diante do contexto pandêmico da COVID-19. REAID [Internet]. 17 ago. 2020 [citado 17 marc. 2021];93:e020005. Available from: https:// www.revistaenfermagematual.com.br/index.php/revista/article/view/774

15 Tilburg TGV, Steinmetz S, Stolte E, Roest HVD, Vries DH. Loneliness and Mental Health During the COVID-19 Pandemic: a study among dutch older adults. J Gerontol B Psychol Sci Soc Sci, 2020, 20(20): 1-7

16 Dubey S, Biswas P, Ghosh R, Chatterjee S, Dubey MJ, Chatterjee S, et al. Psychosocial impact of COVID-19. Diabetes Metab Syndr. 2020; 14(5):779-788.

17 Sociedade Brasileira de Geriatria e Gerontologia (SBGG) [Internet]. Recomendações para Prevenção e Controle de infecções por coronavírus (SARS-Cov-2) em Instituições de Longa Permanência para Idosos (ILPIs). [updated 17 mar. 2020; cited 2020 Dez 17]. Available from: https://sbgg.org.br/recomendacoes-para-prevencao-e-controle-deinfeccoes-por-coronavirus-sars-cov-2-em-instituicoes-de-longa-permanencia-paraidosos-ilpis/

18 Nunes VMA, Machado FCA, Morais MM, Costa LA, Nascimento ICS, Nobre TTX, Silva ME. COVID-19 e o cuidado de idosos: recomendações para instituições de longa permanência. 1st ed. EDUFRN: Natal; 2020

19 Boas P, Wachholz P. Covid-19 em instituições de longa permanência para idosos. Revista Aptare. 2020 Sep 8(36): 24-27 [cited 2020 Dez 17];[about 1 p.]. Available from: http:/ / revistaaptare.com.br/2020/09/16/covid-19-em-instituicoes-de-longapermanencia-para-idosos/ 